\title{
Point-of-care Ultrasound in Anaesthesia and Intensive Care Medicine
}

The use of point-of-care (POC) ultrasonography (US) in anaesthesia and intensive care practice has grown exponentially in the last decades. As US equipment becomes less expensive and more portable, its accessibility and potential applications will continue to grow. Initially adopted as a means of improving the safety and efficacy of therapeutic interventions, POC US has evolved to include monitoring and diagnostic applications in the perioperative and intensive care settings. Although a valuable development, it must be noted that image acquisition and interpretation is user dependent and therefore, each application of US comes with its own risks and benefits. These must be weighed carefully, particularly when treatment decisions are based on sonographic examination.

In terms of therapeutic interventions, ultrasound guided insertion of central venous catheters (CVC) was one of the first uses of US in anaesthesia. This has now become common practice and is recommended by the majority of international guidelines. A Cochrane meta-analysis showed that US guided CVC placement improved the quality and safety of CVC insertion when compared to the traditional landmark technique, reducing overall complication rates and number of attempts taken for insertion, particularly when cannulating the internal jugular vein [1].

Similarly, US guided peripheral nerve blockade has revolutionized regional anaesthesia, not only making it safer and more effective compared to landmark techniques, but also easier to teach and therefore more accessible to novices [2]. Although not widely adopted, US has also been used to aid the administration of neuraxial anaesthesia, particularly as a pre-procedural scan to ascertain the interspinous space [3]. It is possible that some anaesthetists find the use of US for

Address for correspondence:

Gabriella Iohom, MD, PhD Department of Anaesthesia and Intensive Care Medicine Cork University Hospital Wilton Road, Cork, Ireland E-mail: gabriella.iohom@hse.ie neuraxial anaesthesia unnecessary. However, as obesity rates increase, landmark techniques will become more difficult and we may soon be relying on US for neuraxial blockade more than we have in the past. Ultrasound is also widely used in interventional pain medicine and has been shown to be as effective as fluoroscopy for both transforaminal nerve root and facet joint injections [4].

The diagnostic applications of POC US are manifold and still evolving. It is important to note that irrespective of the organ system or body part being examined, ultrasonography is an adjunct to the clinical examination. Typically, it focuses on answering a particular question relevant to the current clinical situation, and does not replace detailed diagnostic imaging interpreted by a radiologist (or cardiologist for echocardiography) [5]. The most common diagnostic application of POC US at present is in the management of undifferentiated shock [6] with a number of protocols published on how to systematically incorporate POC US into the assessment and management of these patients. These protocols include focused cardiac, lung and abdominal US, mainly differing on the priority of the exam sequence [7].

Focused Cardiac Ultrasound (FoCUS) - ascertains the presence or absence of three main findings: pericardial effusion, abnormal left ventricular contractility and right ventricular dilatation as a means of identifying potentially reversible pathophysiology [7]. It is used to answer critical questions in a binary fashion, with no attempt made to measure or quantify pathology [8]. Major valve abnormalities such as severe aortic stenosis or mitral regurgitation can be observed and should prompt referral for more definitive assessment and quantification [9]. Typically, four views are attempted: parasternal long axis, parasternal short axis, subcostal and apical. However, in the critically ill patient, particularly if intubated and ventilated, some views may be unattainable. FoCUS adds to other clinical data in the diagnosis of volume depletion, cardiogenic shock, and pulmonary embolism. There is limited observational 
evidence that the integration of FoCUS into practice improves patient outcomes, but as yet randomized trials are lacking [10]. Identified barriers to the widespread adoption of FoCUS include lack of training, fear of missing diagnoses, lack of resources and a lack of formal accreditation opportunities [11].

Focused Lung Ultrasound (LUS) - adopts a pathophysiological approach to patient assessment [12]. Normal aerated lungs are not visible with US, LUS therefore relies on the recognition of patterns that are pathognomonic for particular disease processes. LUS has excellent sensitivity and specificity in identifying pneumothorax, chronic obstructive pulmonary disease, pneumonia and pulmonary oedema [6]. LUS is included in a number of systematic protocols to aid the assessment of patients in respiratory distress (BLUE protocol) and/ or circulatory collapse (FALLs protocol) [12].

Focused Abdominal US - in anaesthesia and critical care is predominantly used in the assessment of shock. Initially being confined to the Focused Assessment with Sonography in Trauma (FAST scan) which assesses for free fluid in the abdomen as a source of hypovolaemic shock, it has now evolved to include assessment for urinary or biliary obstruction as a reversible source of sepsis. Furthermore, US can be used to examine the aorta for aneurysm in patients presenting with signs of rupture [5]. In addition, gastric ultrasound has been described with measurement of antral cross-sectional area as an indication of increased risk of aspiration at induction of anaesthesia. Confirmation of gastric tube placement by US reduces the need for X-ray exposure in the intensive care unit [4].

Airway Ultrasound - can determine the size of the airway, identify anatomical variations that make tracheal intubation difficult as well as being able to locate the cricoid cartilage in an emergency where front of neck access is required (13).

Optic Nerve Ultrasound - although user specific, measurement of nerve sheath diameter correlates well with intracranial pressure (ICP) [5]. ICP evaluation is important for the anaesthetist in the initial management of patients with traumatic brain injury, and prior to diagnostic dural puncture, or neuraxial anaesthesia. In addition, serial POC US assessments may be considered as monitoring devices in particular for intravascular volume and respiratory function [14].

In summary, US has become an integral part of anaesthesia and intensive care practice, both in the guidance of therapeutic interventions and as a diagnostic aid. POC US should be focused on specific clinical questions and seen as an adjunct to physical examination. It is a relatively inexpensive, safe, noninvasive investigation, but it mandates appropriate training and accreditation before clinical decisions can be based on its use. Given the required economic investment in equipment and training, further investigation of the impact of ultrasound utilization on patient outcomes is important.

\section{David Roche, MD, Gabriella Iohom, MD, $\mathrm{PhD}$}

Department of Anaesthesia and Intensive Care Medicine, Cork University Hospital, Cork, Ireland

\section{Conflict of interest}

Nothing to declare

\section{References}

1. Saugel B, Scheeren TWL, Teboul JL. Ultrasound-guided central venous catheter placement: a structured review and recommendations for clinical practice. Crit Care 2017; 21: 225. doi: 10.1186/s13054-017-1814-y

2. Ranganath A, Srinivasan KK, Iohom G. Ultrasound guided axillary brachial plexus block. Med Ultrason 2014; 16: 246-251. doi: 10.11152/mu.2013.2066.163.2kks

3. Srinivasan KK, Lee PJ, Iohom G. Ultrasound for neuraxial blockade. Med Ultrason 2014; 16: 356-363. doi: 10.11152/ mu.201.3.2066.164.kks1

4. Terkawi AS, Karakitsos D, Elbarbary M, Blaivas M, Durieux ME. Ultrasound for the anesthesiologists: present and future. ScientificWorldJournal 2013; 2013: 683-685. doi: 10.1155/ 2013/683685

5. Wilson S, Mackay A. Ultrasound in critical care. CEACCP 2012; 12: 190-194. doi: 10.1093/bjaceaccp/mks019

6. Diaz-Gomez JL, Via G, Ramakrishna H. Focused cardiac and lung ultrasonography: implications and applicability in the perioperative period. Rom J Anaesth Intensive Care 2016; 23 : 41-54. doi: 10.21454/rjaic.7518.231.lus

7. Seif D, Perera P, Mailhot T, Riley D, Mandavia D. Bedside ultrasound in resuscitation and the rapid ultrasound in shock protocol. Crit Care Res Pract 2012; 2012: 503254. doi: 10.1155/2012/503254

8. Coker BJ, Zimmerman JM. Why Anesthesiologists Must Incorporate Focused Cardiac Ultrasound Into Daily Practice. Anesth Analg 2017; 124: 761-765. doi: 10.1213/ANE. 0000000000001854

9. Haskins SC, Tanaka CY, Boublik J, Wu CL, Sloth E. Focused Cardiac Ultrasound for the Regional Anesthesiologist and Pain Specialist. Reg Anesth Pain Med 2017; 42: 632-644. doi: 10.1097/AAP.0000000000000650

10. Heiberg J, El-Ansary D, Canty DJ, Royse AG, Royse CF. Focused echocardiography: a systematic review of diagnostic and clinical decision-making in anaesthesia and critical care. Anaesthesia 2016; 71: 1091-1100. doi: 10.1111/anae.13525

11. Conlin F, Connelly NR, Eaton MP, Broderick PJ, Friderici J, Adler AC. Perioperative Use of Focused Transthoracic Cardiac Ultrasound: A Survey of Current Practice and Opinion. Anesth Analg 2017; 125: 1878-1882. doi: 10.1213/ANE. 0000000000002089

12. Lichtenstein DA. Lung ultrasound in the critically ill. Ann Intensive Care 2014; 4: 1. doi: 10.1186/2110-5820-4-1

13. You-Ten KE, Siddiqui N, Teoh WH, Kristensen MS. Point-ofcare ultrasound (POCUS) of the upper airway. Can J Anaesth 2018; 65: 473-484. doi: 10.1007/s12630-018-1064-8

14. Mittal AK, Gupta N. Intraoperative lung ultrasound: A clinicodynamic perspective. J Anaesthesiol Clin Pharmacol 2016; 32: 288-297. doi: 10.4103/0970-9185.188824

Rom J Anaesth Intensive Care 2018; 25: $95-96$ 